\title{
A quantitative way to estimate clinical off-target effects for human membrane brain targets in CNS research and development
}

This article was published in the following Dove Press journal:

Journal of Experimental Pharmacology

30 April 2012

Number of times this article has been viewed

\author{
Athan Spiros' \\ Hugo Geerts ${ }^{1,2}$ \\ 'In Silico Biosciences, Berwyn, \\ PA, USA; ${ }^{2}$ School of Medicine, \\ University of Pennsylvania, \\ Philadelphia, PA, USA
}

Correspondence: Hugo Geerts In Silico Biosciences, Inc, 686 Westwind Dr, Berwyn, PA19312, USA

Tel +l 2676798090

Fax + I 7818627690

Email hugo-geerts@in-silico-biosciences. com

\begin{abstract}
Although many preclinical programs in central nervous system research and development intend to develop highly selective and potent molecules directed at the primary target, they often act upon other off-target receptors. The simple rule of taking the ratios of affinities for the candidate drug at the different receptors is flawed since the affinity of the endogenous ligand for that off-target receptor or drug exposure is not taken into account. We have developed a mathematical receptor competition model that takes into account the competition between active drug moiety and the endogenous neurotransmitter to better assess the off-target effects on postsynaptic receptor activation under the correct target exposure conditions. As an example, we investigate the possible functional effects of the weak off-target effects for dopamine-1 receptor $\left(D_{1} R\right)$ in a computer simulation of a dopaminergic cortical synapse that is calibrated using published fast-cyclic rodent voltammetry and human imaging data in subjects with different catechol-O-methyltransferase genotypes. We identify the conditions under which off-target effects at the $\mathrm{D}_{1} \mathrm{R}$ can lead to clinically detectable consequences on cognitive tests, such as the N-back working memory test. We also demonstrate that certain concentrations of dimebolin (Dimebon), a recently tested Alzheimer drug, can affect $D_{1} R$ activation resulting in clinically detectable cognitive decrease. This approach can be extended to other receptor systems and can improve the selection of clinical candidate compounds by potentially dialingout harmful off-target effects or dialing-in beneficial off-target effects in a quantitative and controlled way.
\end{abstract}

Keywords: off-target pharmacology, working memory, dopamine, COMT

\section{Introduction}

The selection of a compound for clinical development in central nervous system (CNS) diseases is based on the specific pharmacology aimed at rodent targets and the subsequent demonstration of activity in a preclinical animal model, which usually takes the form of a dose-dependent reduction of the dysfunctional behavior or a neuropathological biomarker.

The failure rate of CNS drugs in clinical development is still very high (over $90 \%),{ }^{1}$ meaning that less than one out of ten drugs that enter Phase I studies reach the market. However, all of these drugs are found to be safe and efficacious in at least a few preclinical animal models. The reasons for this translational disconnect include (1) differences in neurotransmitter circuitry and drug metabolism, (2) the incomplete representation of the full human pathology in animal models, (3) the absence of important functional genotypes in animal models, (4) the pharmacodynamic interference of allowed comedications, (5) the difference in drug affinities between rat and human 
subtype receptors, and (6) the underestimation of off-target effects. $^{2}$

While all of the reasons mentioned above contribute to possible failures in the clinical setting, the issue of offtarget effects can be addressed relatively easily in early preclinical drug discovery. Usually, the clinical candidate is screened against a number of other receptors, and offtarget interference is often estimated based upon the ratio of binding affinities. This approach fails to take into account the relative affinity of the endogenous neurotransmitter and the drug at the off-target receptor, and the actual drug exposure in the brain.

This report addresses the issue of off-target activities of clinical candidates and presents evidence that the estimation of these effects using drugs' ratios of affinity values on the primary versus secondary targets can lead to erroneous conclusions in the clinical setting.

We use a quantitative systems pharmacology approach which consists of a computational model of a brain synapse that simulates the competition of the neurotransmitter and different agents against the same postsynaptic receptorbinding site. This model is based on realistic biological firing frequencies with a presynaptic membrane, which contains negative feedback autoreceptors and incorporates a firing frequency-dependent neurotransmitter release.

To achieve a better estimate of the clinical effects of such differences, we simulated the effect of small changes in dopamine- 1 receptor $\left(D_{1} R\right)$ inhibition and compared these changes to situations with clinically detectable differences on neuropsychological scales in working memory.

Dopamine $\mathrm{D}_{1} \mathrm{R}$ activation in the cortex is known to influence working memory in humans ${ }^{3}$ and is modulated by levels of free dopamine. Cortical dopamine clearance is predominantly regulated by catechol-O-methyltransferase (COMT), an enzyme that breaks down dopamine and norepinephrine. ${ }^{4}$ Subjects with Val158Val genotype of the COMT enzyme have higher enzymatic activity and function consistently worse on clinical scales of working memory compared to subjects with the Met158Met genotype. 5,6

The dynamics of this computer model are calibrated using published experimental high-resolution data on free cortical dopamine levels in rodents and are constrained by imaging studies in human subjects. We further calibrate the effect of the COMT genotypes on cortical synaptic dopamine half-life using PET-imaging data $^{7}$ on the binding potential of the NNC-112 radiotracer to the cortical $\mathrm{D}_{1}$ receptor in healthy subjects. The in silico simulation model enables us to compare the differences in human $\mathrm{D}_{1} \mathrm{R}$ activation as a consequence of off-target effects with the $\mathrm{D}_{1} \mathrm{R}$ activationlevel differences between different COMT genotypes. This data can potentially identify clinical liabilities regarding cognition.

\section{Methods \\ Drug affinities}

The National Institute of Mental Health's Psychoactive Drug Screening Program, Contract \# NO1MH32004 (NIMH PDSP) generously provided the $\mathrm{K}_{\mathrm{i}}$ determinations and receptor binding profiles of specific drugs. The NIMH PDSP is directed by Bryan L Roth $\mathrm{MD}, \mathrm{PhD}$ at the University of North Carolina at Chapel Hill and Project Officer Jamie Driscol at NIMH, Bethesda MD, USA (http://pdsp.med.unc. edu/indexR.html).

\section{Receptor competition model}

The receptor competition model is a set of one-dimensional temporal differential equations that simulate in a biophysically realistic synaptic physiology the competition of neurotransmitters and up to three different agents for binding sites on pre- and postsynaptic receptors, and is based on the relative affinities and concentrations of each of the different agents. ${ }^{8}$ The model simulates the release of neurotransmitters following a userdefined set of firing patterns and is modulated by the physiological activity at the presynaptic autoreceptor and a depression or facilitation mechanism. The half-life of the neurotransmitter in the synaptic cleft is determined by the removal of the neurotransmitter by transporters (such as the dopamine transporter) or enzymes (such as COMT), as well as diffusion. Although the model does not include detailed molecular intracellular simulations, it uses phenomenological equations that can be calibrated using experimental data.

The model is calibrated using published experimental fast-cyclic voltammetry data on the cortical dopaminergic synapse in rodents and confirmed with published imaging data in humans. In this synapse, dopamine interacts with presynaptic $\mathrm{D}_{2}$ autoreceptors in a negative feedback cycle and with postsynaptic $\mathrm{D}_{1}$ receptors. We assume an affinity for dopamine of $10 \mathrm{nM}$ for the $\mathrm{D}_{2} \mathrm{R}^{9,10}$ and $130 \mathrm{nM}$ for the $\mathrm{D}_{1} \mathrm{R}^{11}$

In contrast with the striatum where dopamine removal is driven by the dopamine transporter (DAT) mediated uptake, cortical dopamine clearance is also driven by the COMT, ${ }^{4}$ which leads to a 5-8-fold longer DA half-life compared to the $\mathrm{N}$ accumbens. ${ }^{12}$ 


\section{Mathematical description of the generic receptor competition model}

This section presents a detailed description of the receptor competition model.

Dynamical binding and unbinding of the different agents to the receptor sites are calculated as follows. If [dop] is the free DA concentration and $\left[\mathrm{D}_{\mathrm{f}}\right]$ is the concentration of free receptors, then the change in receptors bound by dopamine $\left[D_{n}\right]$ is governed by the following ordinary differential equation (ODE):

$$
\partial\left[\mathrm{D}_{\mathrm{n}}\right] / \partial \mathrm{t}=\mathrm{k}_{\mathrm{on}}^{\mathrm{n}} \times[\mathrm{dop}] \times\left[\mathrm{D}_{\mathrm{f}}\right]-\mathrm{k}_{\mathrm{on}}^{\mathrm{n}} \times \mathrm{K}_{\mathrm{d}}^{\mathrm{n}} \times\left[\mathrm{D}_{\mathrm{n}}\right]
$$

with the initial condition that all receptors begin in the free state (subscript n refers to the "neurotransmitter" dopamine). With sub- and superscripts $\mathrm{n}, \mathrm{d}, \mathrm{m}$, and t referring to neurotransmitter, drug, metabolite, and tracer, respectively, the change in receptors for drug, metabolite and tracer binding is governed by the following coupled ODEs:

$$
\begin{aligned}
& \partial\left[\mathrm{D}_{\mathrm{d}}\right] / \partial \mathrm{t}=\mathrm{k}_{\mathrm{on}}^{\mathrm{d}} \times[\mathrm{drug}] \times\left[\mathrm{D}_{\mathrm{f}}\right]-\mathrm{k}_{\mathrm{on}}^{\mathrm{d}} \times \mathrm{K}_{\mathrm{d}}^{\mathrm{d}} \times\left[\mathrm{D}_{\mathrm{d}}\right] \\
& \partial\left[\mathrm{D}_{\mathrm{m}}\right] / \partial \mathrm{t}=\mathrm{k}_{\mathrm{on}}^{\mathrm{m}} \times[\text { met }] \times\left[\mathrm{D}_{\mathrm{f}}\right]-\mathrm{k}_{\mathrm{on}}^{\mathrm{m}} \times \mathrm{K}_{\mathrm{d}}^{\mathrm{m}} \times\left[\mathrm{D}_{\mathrm{m}}\right] \\
& \partial\left[\mathrm{D}_{\mathrm{t}}\right] / \partial \mathrm{t}=\mathrm{k}_{\mathrm{on}}^{\mathrm{t}} \times[\text { tracer }] \times\left[\mathrm{D}_{\mathrm{f}}\right]-\mathrm{k}_{\mathrm{on}}^{\mathrm{t}} \times \mathrm{K}_{\mathrm{d}}^{\mathrm{t}} \times\left[\mathrm{D}_{\mathrm{t}}\right]
\end{aligned}
$$

Alternatively, the metabolite can be substituted for a second drug in polypharmacy simulations. In general, $\mathrm{K}_{\mathrm{d}}=\mathrm{k}_{\mathrm{off}} / \mathrm{k}_{\mathrm{on}}$. Furthermore,

$$
\mathrm{D}_{\mathrm{f}}=\mathrm{D}_{\mathrm{o}}-\mathrm{D}_{\mathrm{n}}-\mathrm{D}_{\mathrm{d}}-\mathrm{D}_{\mathrm{m}}-\mathrm{D}_{\mathrm{t}}
$$

where $\mathrm{D}_{\mathrm{o}}$ is the concentration of receptors.

The amount of free dopamine depends on two processes: exponential decay and quantal release. Exponential decay is classically defined as $[\mathrm{dop}](\mathrm{t})=\exp (-\mathrm{t} \ln (2) /$ half-life $)$ where half-life is the half-life of the decay process. At times of release, [dop] is immediately updated by adding the release amount. ${ }^{13}$

Therefore, the amount of presynaptic receptor activation that occurred $150 \mathrm{~ms}$ before the current release event determines the amount of new release as follows:

$$
=\operatorname{release}_{0}\left[1+\operatorname{relScale}\left(1-2 \frac{\operatorname{recAct}^{\text {relsens }}}{\text { recAct }^{\text {relsens }}+\text { normBound }^{\text {relSens }}}\right)\right]
$$

where release ${ }_{0}$ is the base release amount, relScale is the maximum relative change for release, recAct is the receptor activation at the specified time in the past, relSens is the sensitivity to the presynaptic receptor (lower values create a shallow response and higher values create a sharp difference between activation levels), and normBound is the amount of normal presynaptic binding that one would expect in the tonic case (ie, when recAct equals normBound, the new release equals the baseline release amount). The parameters are calibrated so that the coupling of presynaptic $\mathrm{D}_{2} \mathrm{R}$ activation to dopamine release reflects the actual experimental data (as seen in the Results section). All differential equations are solved with a fourth-order Runge-Kutta method with a time step of $0.01 \mathrm{msec}$.

In addition, the release can be modulated by a depression or facilitation mechanism. ${ }^{14}$ Instead of using internal $\mathrm{Ca}^{++}$levels to determine dopamine release, we consider the facilitation and depression of dopamine release based solely on the amount of time elapsed since the previous firing using a phenomenological equation. Therefore, the amount of dopamine released is based on the history of firing as well as the activation level of the presynaptic $\mathrm{D}_{2}$ autoreceptors. If we denote the time of the nth firing by $t_{n}$, then the release amount is modified based on all previous firings as follows:

$$
\begin{aligned}
& \text { release }_{\text {new }} \\
& =\operatorname{release}\left(1+\sum_{i=1}^{n-1} w_{f} \exp \left[-k_{f}\left(t_{n}-t_{i}\right)\right]-w_{d} \exp \left[-k_{d}\left(t_{n}-t_{i}\right)\right]\right)
\end{aligned}
$$

where $\mathrm{w}_{\mathrm{f}}$ is the facilitation weight, $\mathrm{w}_{\mathrm{d}}$ is the depression weight, $\mathrm{k}_{\mathrm{f}}$ is the decay rate of facilitation, and $\mathrm{k}_{\mathrm{d}}$ is the decay rate of depression. The Results section shows how these parameters are calibrated to the experimental data.

The simulation is initiated by finding the equilibrium given a constant amount of free dopamine at $500 \mathrm{nM}$. The simulation is then run for a transitory time of 5 seconds at the tonic-firing rate of $4 \mathrm{~Hz}$. Finally, the simulation runs for an additional 2.5 seconds during which the average binding levels are determined and the simulation is ready to compute the task.

This approach is completely deterministic, given a predefined stimulation paradigm. However, specific parameter changes - as a consequence of genotypes in certain patient populations - in biological processes of this generic synapse might lead to different outcomes. Finally, the module is programmed in Java and implemented both as an interactive graphical user interface and as a batch file that allows multiple simulations. 


\section{Results}

\section{Calibration of a cortical dopaminergic} synapse

This paper focuses on cortical dopamine $\mathrm{D}_{1} \mathrm{R}$ receptor activation levels because primate data have suggested that this is a key receptor in working memory ${ }^{15}$ and that changes in dopamine dynamics, such as those caused by the presence of different genotypes of the COMT enzyme, have been documented as clinically detectable in tests of working memory. ${ }^{5}$

Unlike in the primate striatum, ${ }^{16}$ there are no experimental fast cyclic voltammetry data available on actual cortical dopamine dynamics in the primate, so we calibrated the computer model of the cortical dopaminergic synapse using existing rapid-cyclic voltammetry data on free dopamine in rodents in vivo. ${ }^{12}$ In this experiment, dopamine levels were measured in nine different conditions of forced firing frequency of VTA neurons or with different stimulus durations at $50 \mathrm{~Hz}$. The different parameters describing the cortical presynaptic $\mathrm{D}_{2} \mathrm{R}$ autoreceptor physiology in the computer model were adjusted to fit the experimental data (see Figure 1). The release of dopamine is five times lower, compared to the calibration of the rodent striatal dopaminergic synapse, although the coupling strength of the presynaptic $\mathrm{D}_{2} \mathrm{R}$ autoreceptor activation to presynaptic DA release is similar. ${ }^{8}$

A

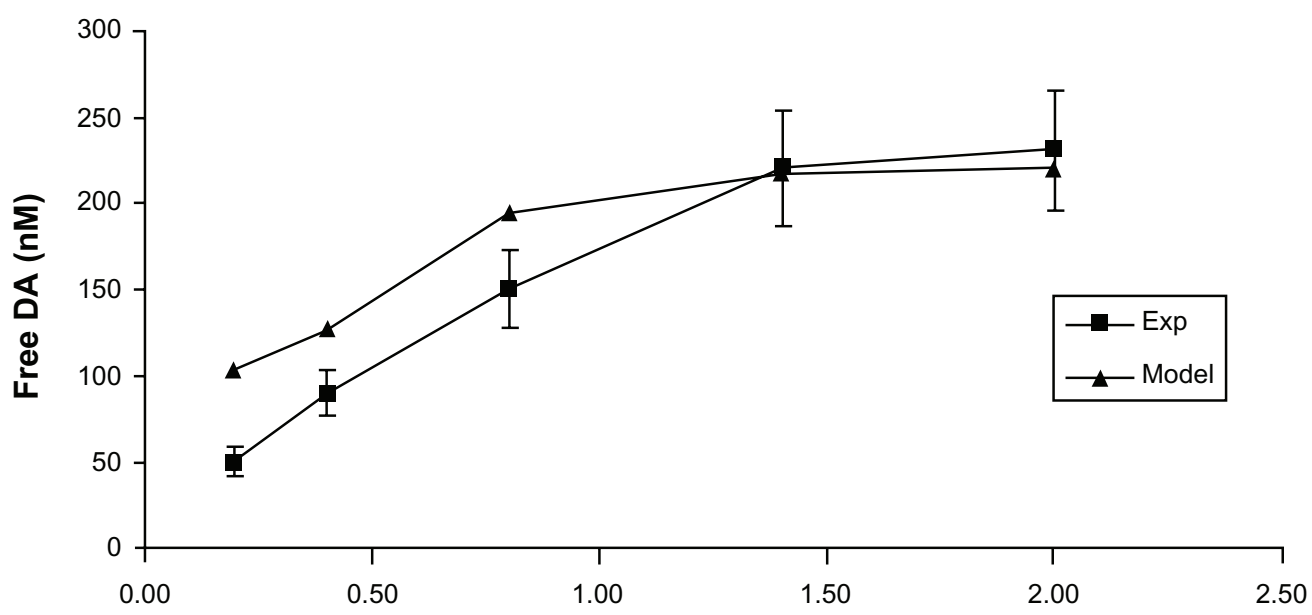

Stimulus duration (sec)

B

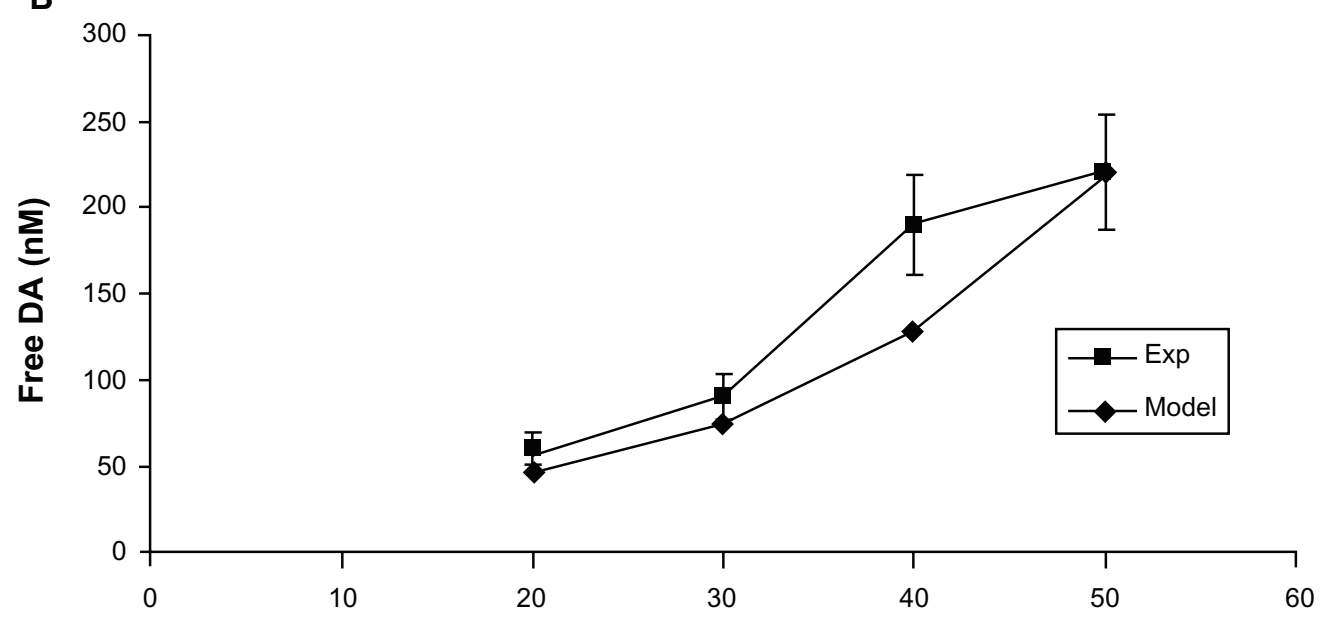

Frequency stimulus $(\mathrm{Hz})$

Figure I Calibration of the receptor competition model for the cortical dopaminergic synapse with experimental data in the rodent. ${ }^{2}$ Free DA is measured with rapid cyclic voltammetry under conditions of forced-firing of the ventral tegmentum area, either at different frequencies $(\mathbf{A})$ or for different durations (B) at a fixed frequency of $50 \mathrm{~Hz}$. Alignment of the experimental data with the model data output allows us to define the specific parameters for presynaptic $\mathrm{D}_{2}$ autoreceptor coupling, facilitation, and depression of synaptic release based on the previous firing history and half-life of dopamine in the synaptic cleft for the receptor competition model.

Abbreviations: DA, dopamine. 


\section{Calibrating the cortical dopaminergic synapse for the different COMT genotypes}

The effect of the different Catechol-O-Methyl Transferase (COMT) genotypes on dopamine dynamics has been studied in humans using the displacement of ${ }^{11} \mathrm{C}-\mathrm{NNC}-112$, a $\mathrm{D}_{1} \mathrm{R}$ specific radiotracer in the human cortex. ${ }^{7}$ Using these data, we can implement the effect of the COMT genotype on dopamine half-life.

The authors reported changes in the binding potential between genotypes, with the smallest difference in absolute binding potential $(0.12)$ reported in the temporal cortex and the largest difference $(0.36)$ reported in the occipital cortex. In all these areas, the binding potential rank order was Val-Val (VV) $>$ Met-Val (MV) > Met-Met (MV), which is in line with the expected higher dopamine concentration in Met-Met carriers as a consequence of a lower enzymatic activity and catecholamine breakdown (ie, more dopamine competes with the tracer, which leads to less tracer binding) ${ }^{17}$ Average differences in the absolute binding potential were 0.15 between MV and $\mathrm{VV}$ and 0.22 between MM and VV. With an average reported binding potential of 0.75 for the $\mathrm{VV}$ genotype, this yielded normalized changes of $20 \%(0.15-0.75)$ for the MV-VV difference and 29\% (0.22-0.75) for the MM-VV difference.

Such a result can be interpreted as an upregulation of $D_{1} R$ density in the $\mathrm{V} / \mathrm{C}$ carriers or as a decrease in DA levels in the $\mathrm{V} / \mathrm{V}$ that allow more tracer to bind. Assuming that the majority of this effect is due to lower DA levels, we can identify what changes in DA half-life would correspond to the reported changes in imaging-binding potential.

In order to translate this binding potential into actual receptor occupancies, we assume that the normalized change in binding potentials is proportional to the normalized difference in tracer binding to the receptor (all normalizations are against the Val-Val case). We also use published affinity data for the NNC-112 tracer of $0.18 \mathrm{nM}$ for $\mathrm{D}_{1}$, and $898 \mathrm{nM}$ for $\mathrm{D}_{2}$.

The half-life for DA is systematically changed in the model dopaminergic synaptic cleft with presynaptic $\mathrm{D}_{2}$ autoreceptors, postsynaptic $\mathrm{D}_{1}$ receptors, and dopaminergic tonic-burst firings of 4,40 , or $80 \mathrm{~Hz}$, respectively. The imaging data in humans were best approximated with a change in cortical DA half-lives from $110 \mathrm{msec}$ in the Val-Val case, to $200 \mathrm{msec}$ in the Met-Val case, to $300 \mathrm{msec}$ in the Met-Met case (see Figure 2).

When these values are applied to a normal baseline situation, $\mathrm{D}_{1} \mathrm{R}$ activation levels (the average proportion of $\mathrm{D}_{1} \mathrm{R}$ bound by dopamine) decline from $30.95 \%$ in the $\mathrm{MM}$ case, to $27.9 \%$ in the MV case, and to $23.57 \%$ in the VV case. Therefore, the $\mathrm{D}_{1} \mathrm{R}$ in the MM case is $11 \%$ more active than in the MV case, and 31\% more active than in the VV case.

\section{Further support for the model of a cortical dopaminergic synapse}

Because the previous calibration of the cortical dopaminergic synapse was performed using experimental rodent data,

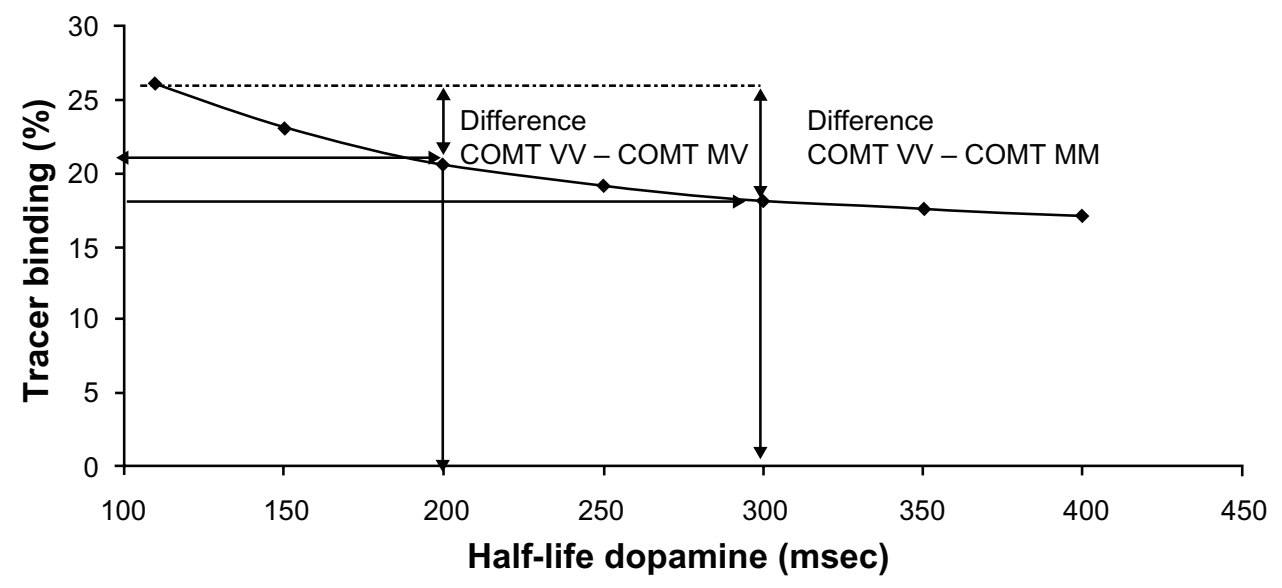

Figure 2 Results from the receptor competition model simulation suggest that the PET imaging data in subjects with different COMT Vall58/Met genotypes on the displacement of the $D_{1} R$ specific radiotracer, NNCI 12, by endogenous dopamine ${ }^{7}$ are best represented by the modulation of cortical synaptic DA half-life. The changes in radiotracer binding potential as a consequence of increased free synaptic dopamine correspond to a change in dopamine half-life from II 0 msec in the $\mathrm{Val}-\mathrm{Val}$ case to $200 \mathrm{msec}$ in the $\mathrm{Val}-\mathrm{Met}$ case (relative tracer binding decreasing from 0.260 to 0.206 [a $20 \%$ decrease] corresponds to a change in receptor binding from $30.9 \%$ to $27.9 \%$ ), and $300 \mathrm{msec}$ in the Met-Met case (relative tracer binding decreasing from 0.26 to 0.18 corresponds to a decrease in receptor binding from $30.9 \%$ to $23.6 \%$, a change of $30 \%$, see vertical arrow).

Abbreviations: COMT MM, Catechol-O-Methyl Transferase Met/Met; COMT MV, Catechol-O-Methyl Transferase Met/Val; COMT VV, Catechol-O-Methyl Transferase $\mathrm{Val} / \mathrm{Val}$; DA, dopamine; PET, positron emission tomography. 
we wanted to test whether the model output would correspond at least qualitatively to results from humanimaging data on changes in cortical dopamine levels.

The COMT genotype has no effect on the basal occupancy level of FLB457, a specific radiotracer for $\mathrm{D}_{2} \mathrm{R}$ in nonmedicated humans. ${ }^{18}$ The model outcome suggests that the fraction of FLB457 bound to (presynaptic) $\mathrm{D}_{2} \mathrm{R}$ changes from $4.8 \%$ to $5.8 \%$ in COMT Met-Met carriers to Val-Val carriers in a regular dopaminergic-firing pattern. It is likely that this small difference is beyond experimental detection.

Recent studies using repetitive transcranial magnetic stimulation (rTMS) using a $10 \mathrm{~Hz}$ stimulus train suggest an increase in cortical dopamine as measured by a decrease in the binding of FLB417. ${ }^{19}$ Cortical afferent fibers regulate the firing of VTA DA neurons; therefore, we simulate this clinical experiment by assuming that the dopaminergic afferent firing to the cortex is also forced to the same frequency of $10 \mathrm{~Hz}$. Compared to a regular tonic-burst dopaminergic firing pattern (consisting of tonic epochs of $4 \mathrm{~Hz}$, interspersed with a short burst firing of $40-80 \mathrm{~Hz}$, see above), the simulations suggest that a dopaminergic synapse with the COMT Met-Met half-life has a $55 \%$ decrease in $\mathrm{D}_{2} \mathrm{R}$ binding of FLB457, while the COMT Met-Val and COMT Val-Val parameter sets have a $56 \%$ and a $58 \%$ decrease in binding, respectively. Because the subjects in the rTMS experiment were not genotyped, we assumed a weighted $56 \%$ decrease in FLB457 binding potential, which is slightly higher than the reported $37 \%$ decrease in the subgenual and the $45 \%$ decrease in the pregenual anterior cingulate. ${ }^{19}$

\section{Effect of different drug affinities on dopamine $D$, receptor activation}

In preclinical drug discovery, off-target effects of candidate compounds are often qualitatively estimated using ratios of affinities against primary and secondary targets. This might lead to erroneous conclusions because the endogenous activity of the neurotransmitter for the secondary target can be much weaker than the affinity of the substrate to the primary target, which leads to relatively greater displacement by the drug.

As an example, we simulated the effect on $D_{1} R$ activation levels of a hypothetical drug with a range of affinities between 80 and $1280 \mathrm{nM}$ for the human $\mathrm{D}_{1} \mathrm{R}$ in COMT Met-Met subjects. Figure 3 shows a number of conditions that might result in $\mathrm{D}_{1} \mathrm{R}$ activations of the same size as COMT MM vs COMT VV subjects and can lead to clinically detectable readouts. For instance, at a concentration of $200 \mathrm{nM}$, a drug with a $\mathrm{K}_{\mathrm{i}}$ of $320 \mathrm{nM}$ in a COMT MM subject leads to a D1R activation level well below a COMT VV subject, while the same concentration of a drug with a $\mathrm{K}_{\mathrm{i}}$ of $1280 \mathrm{nM}$ (fourfold weaker) decreases the $\mathrm{D}_{1} \mathrm{R}$ activation to the level of a COMT MV subject.

Similarly, a drug with a low affinity of $640 \mathrm{nM}$ for the human $\mathrm{D}_{1} \mathrm{R}$ at a functional brain concentration of about $300 \mathrm{nM}$, when given to COMT MM subjects, can decrease the $\mathrm{D}_{1} \mathrm{R}$ activation level to the equivalent of a VV subject. Such an off-target effect can be detected clinically and can have important consequences if the primary indication of the investigative drug is cognitive enhancement. Further simulations suggest

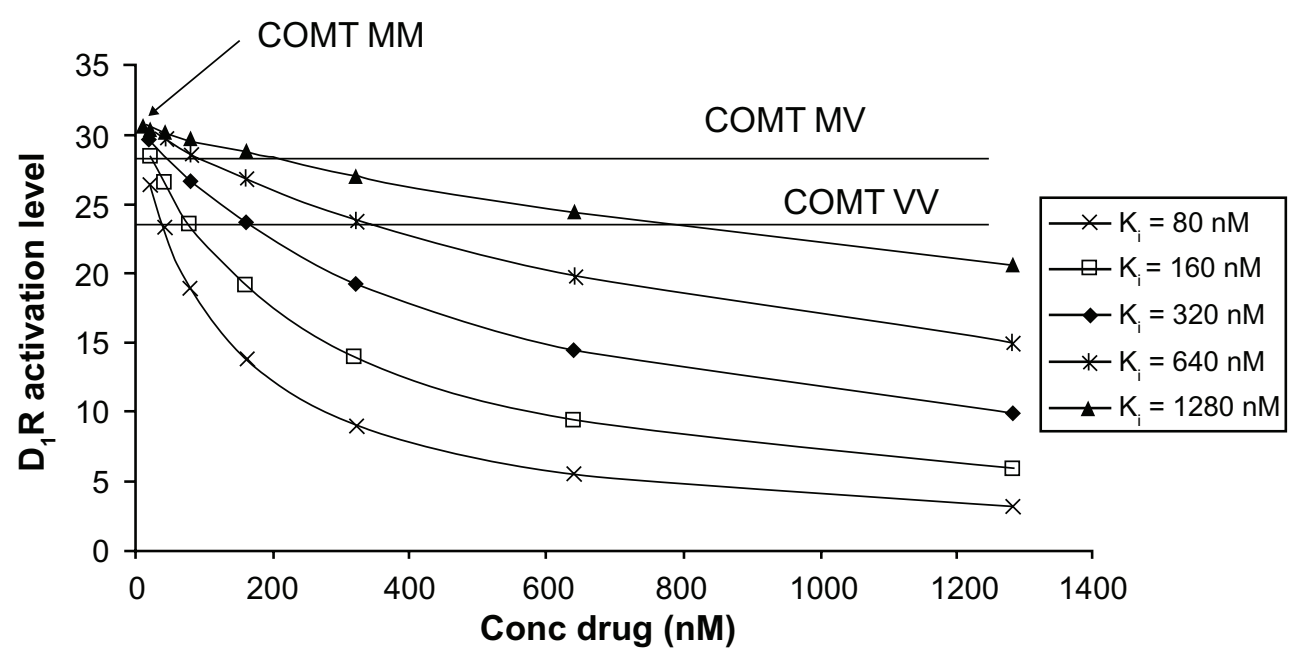

Figure 3 Activation level of $D, R$ (in \%) in a cortical COMT Met/Met environment with full antagonists at different affinities for the $D_{1} R$ (range $80-1280 \mathrm{nM}$ ) and at different concentrations. $D_{1} R$ activation levels corresponding to a COMT Val-Val and COMT Val-Met case are shown as horizontal lines. At a functional brain concentration of $300 \mathrm{nM}$, an antagonist with a $\mathrm{K}_{\mathrm{i}}$ of $640 \mathrm{nM}$ in a COMT MM subject results in a $\mathrm{D}_{1} \mathrm{R}$ activation (23\%) similar to a COMT VV genotype. For a compound with twofold lower affinity $\left(K_{i}=1280 \mathrm{nM}\right)$, this decrease is reached at a functional concentration of $600 \mathrm{nM}$. Such a difference is similar to the differences for the COMT genotypes and can lead to clinically detectable differences on working memory.

Abbreviations: COMT MM, Catechol-O-Methyl Transferase Met/Met; COMT MV, Catechol-O-Methyl Transferase Met/Val; COMT VV, Catechol-O-Methyl Transferase $\mathrm{Val} / \mathrm{Val}$; DA, dopamine. 
that such clinically relevant differences can be detected in weak antagonists for functional doses that are roughly half their $\mathrm{K}_{\mathrm{i}}$ for the neurotransmitter receptor.

In this context, it worth noting that Dimebon (latrepirdine), a compound developed by Medivation (San Francisco, CA, USA) that recently failed in a Phase III trial in Alzheimer's disease, had an affinity of about $600 \mathrm{nM}$ for the human $\mathrm{D}_{1} \mathrm{R}^{20}$ while animal studies show that brain levels of $170 \mathrm{nM}$ and higher can easily be attained. ${ }^{21}$ Our calculations show that this pharmacological activity in a COMT MM subject can decrease $\mathrm{D}_{1} \mathrm{R}$ activation from $31 \%$ to $27 \%$, a level lower than a COMT MV subject (27.9\%), while a $\mathrm{D}_{1} \mathrm{R}$ activation level corresponding to a COMT VV subject is reached at a Dimebon concentration of $320 \mathrm{nM}$. If we assume that the relative difference in working memory observed between schizophrenic COMT MM and VV subjects would hold in Alzheimer patients, these data suggest that Dimebon's $D_{1} \mathrm{R}$ antagonism can significantly reduce the clinical cognitive signal, all other things remaining equal. It is also of interest to note that the $\mathrm{D}_{1} \mathrm{R}$ activation level is COMT-dependent and imbalances between the treatment arms in clinical trials can further affect the cognitive read-out.

This example is specifically provided for cortical $D_{1} R$ activation because the effects can be appreciated in working memory cognitive tasks; however, in a general sense, this approach can also be applied to other receptor systems.

\section{Discussion}

This study aims to estimate the effects of off-target pharmacology for candidate drugs in a quantitative way by using a calibrated computer model to simulate the interaction between the drug and neurotransmitter in humanized synaptic environments. Although this is generally true for any receptor system, this report applies this knowledge to the cortical dopaminergic system. The results of this study suggest that consideration of the affinity of candidate drugs to human receptor subtypes, both as primary or off-target pharmacology, is crucial in candidate drug selection. In some conditions, even modest affinities against the human $\mathrm{D}_{1} \mathrm{R}$ might substantially reduce the signal in clinical cognitive outcomes. In addition to the ratio of affinities for the primary versus the off-target receptors, the off-target effect also depends on the absolute free-brain concentration of the compound and the affinity of the candidate drug in relation to the endogenous affinity of dopamine for the $D_{1} R$.

The dopaminergic cortical synapse is first calibrated using rodent preclinical data. Therefore, the model assumes that the rodent cortical dopaminergic synapse is identical to the human cortical synapse. This is not always the case, as documented in the case of the striatal rodent versus primate synapse,${ }^{8}$ where differences in species might account for the substantial clinical difference between partial $\mathrm{D}_{2} \mathrm{R}$ agonists in schizophrenia. However, the predicted changes in dopamine tracer bindings in humans are qualitatively in line with the reported clinical experiments.

The model also uses a phenomenological approach to describe the coupling of the physiological effect of the presynaptic autoreceptor with subsequent neurotransmitter release, or the impact of firing-history on the future synaptic vesicle release. While this might be sufficient for a number of questions in pharmaceutical CNS research and development, a more comprehensive simulation of intracellular physiology might improve the model, especially when a possible drug target is associated with the biology of these processes.

The calibration using COMT genotypes-related changes in $\mathrm{D}_{1} \mathrm{R}$ imaging ${ }^{7}$ allows us to determine the change in the $\mathrm{D}_{1} \mathrm{R}$ activation level that is associated with the genotypemediated, clinically measurable impact on cognitive scales. ${ }^{5,6}$ This study assumes that the observed changes in binding potential for NNC-112 at the $\mathrm{D}_{1} \mathrm{R}$ are driven by changes in endogenous dopamine tone. Because upregulation of postsynaptic receptors as a consequence of lower DA tone can also contribute to the observed changes in binding potential, the determined dopamine half-life modifications are likely to be somewhat high. If at all, this amplifies the message that off-target effects at the $\mathrm{D}_{1} \mathrm{R}$ can lead to reduced cognitive performance.

Plasma pharmacokinetic profiles do not always reflect the free functional brain concentration of CNS-active drugs; for instance, risperidone and olanzapine have a five- to tenfold longer half-life in the brain, compared to plasma. ${ }^{22}$ Other reports indicate that a free-functional fraction of drugs in the brain can sometimes differ from the total free drug due to nonspecific binding to a lipophilic environment. ${ }^{23}$ Some of these outstanding questions can be addressed using tracer displacement studies in humans, which have the advantage of being able to probe functional intrasynaptic concentrations.

The simulation results suggest that great care must be taken to determine the off-target effects when choosing clinical candidates. Ideally, one should take into account both the affinity of the drug and the neurotransmitter at the off-target receptors, in addition to the functional free drug concentration levels that can be achieved clinically. For instance, suppose a hypothetical candidate disease-modifying agent in Alzheimer's disease has an affinity of $1 \mathrm{nM}$ for its primary intracellular target and an affinity of $640 \mathrm{nM}$ for the dopamine $\mathrm{D}_{1} \mathrm{R}$ (600-fold "selectivity"). In clinical trials, one aims to achieve the drug concentrations that give good 
inhibition levels of the primary target. If the total free and bound drug brain concentration reaches a few hundred nM with the bound drug residing primarily in lipid membranes, this might result in substantially more $\mathrm{D}_{1} \mathrm{R}$ blockage due to a close interaction with membrane-bound receptors. This, in turn, can lead to less than robust effects on functional cognitive scales and to a reduced signal in clinical trials, sometimes leading to the demise of the clinical development project.

When clinical tracer-displacement imaging is applied to the primary target, high drug concentrations in the $>90 \%$ displacement range are often difficult to determined exactly due to the saturation. For example, at clinically relevant doses, the antipsychotic drug, aripiprazole, can easily achieve central $\mathrm{D}_{2}$ receptor occupancies of $94 \%$ and higher, ${ }^{24}$ corresponding to functional drug concentrations over $100 \mathrm{nM}$, some 30 -fold greater than its affinity for the primary target. This can lead to larger-than-anticipated free drug levels that can give rise to substantial off-target effects.

In the case of Alzheimer's disease, this report suggests that the off-target effect of Dimebon at the $\mathrm{D}_{1} \mathrm{R}$ could have contributed to the negative clinical outcome in the Phase III Alzheimer trial. ${ }^{25}$ The model also suggests that the $\mathrm{D}_{1} \mathrm{R}$ activation level is dependent upon the COMT genotype, which is not usually monitored in clinical trials of cognitive deficits. The imbalance of this genotype between treatment arms might add to the variability of the clinical outcome.

However, proper interpretation of the results with Dimebon relies on a number of assumptions. First, we assume that Dimebon has no major pharmacologically active metabolites that could alter the effect on cognitive outcomes. Second, we have no indication for the level of target engagement in clinically relevant doses of Dimebon. Preclinical animal studies indicate that it is possible to reach levels in the hundreds of nM, but human data are lacking. Third, this report only studies the effect of Dimebon on the dopamine $\mathrm{D}_{1} \mathrm{R}$; however, it is known that the compound interacts with many other adrenergic, serotonergic, and cholinergic receptors ${ }^{20}$ that might affect the cognitive outcome. Therefore, the results presented here cannot answer the question of what determines the clinical outcome of Dimebon. Its only purpose is to identify the $\mathrm{D}_{1} \mathrm{R}$ antagonism as a possible liability factor, even though the ratio of affinity for the histamine $H_{1} R$ over this off-target effect was well over 200.

The computer model is based on the cortical dopaminergic synapse, whose kinetics is calibrated with experimental rodent data. The half-life for dopamine that best fits both rodent cyclic voltammetry and human imaging data is in the range of 110-300 msec. This value is in line with the reported
Michaelis-Menten COMT enzyme kinetics with $\mathrm{V}_{\max }$ of about $450 \mathrm{fM} / \mathrm{min} / \mathrm{mg}$ protein. ${ }^{26}$ A molecular weight of 25,000 for the enzyme is used, which corresponds to the conversion of about $10^{9}$ substrate molecules per second for every $1.6 \times 10^{8}$ enzyme molecules, and results in approximately six dopamine molecules/enzyme/second, or a clearance time in the 150-200 msec range. Additionally, the calibration data results in a relative difference of only $11 \%$ in $D_{1} R$ activation between COMT-MM and COMT-MV subjects, but reaches $30 \%$ between MM and VV-COMT subjects. This might explain some of the observations that the clinical difference in cognitive tasks is most pronounced between $\mathrm{MM}$ and $\mathrm{VV}$ carriers, but not as much between MM and MV carriers. ${ }^{27}$ The COMT enzyme affects the dopamine clearance as well as the norepinephrine clearance. ${ }^{26}$ This report assumes that the effect on cognitive performance was driven primarily by cortical dopamine, and much less by cortical norepinephrine. ${ }^{28}$

The model calculations suggests that for certain doses $\mathrm{D}_{1} \mathrm{R}$ affinities in the range of $300-600 \mathrm{nM}$ can have important consequences when considering their effect in realistic CNS synapses, where the endogenous neurotransmitter is present and the drug competes with the neurotransmitter for the same binding site. These differences can lead to a clinically observable cognitive difference as is seen between human COMT Val158Val and Met158Met genotypes. ${ }^{5,6}$

Although these results and discussion might argue for the development of a very selective and potent drug, whereas a multitarget pharmacology is generally preferred for addressing complex CNS disorders. ${ }^{29,30}$ The major challenge is to identify the off-target effects that can improve the symptomatic outcome; indeed, a molecule with both disease-modifying and symptomatic properties in neurodegenerative diseases can have a tremendous advantage in clinical development.

In summary, this report suggests a quantitative way of determining off-target effects, especially in relation to clinical trial outcome in cognitive disorders with regard to the $D_{1} R$. The approach can be applied to a wide variety of receptors and targets. Off-target effects can sometimes lead to an unexpected lower clinical signal that must be studied carefully. Fortunately, this issue can be addressed easily during the early stages of drug discovery. Compared to the total cost of clinical development, determining the full human receptor profile of a candidate drug is inexpensive in the early stages of drug discovery. The extra costs associated with this screening and the careful consideration of these effects through simulation at such an early stage, are minor compared to the large costs of detecting these unfortunate differences later in the clinical development program. 


\section{Acknowledgments}

We thank Patrick Roberts and John Dani for their valuable discussions and comments on the manuscript. This work was supported by In Silico Biosciences. Athan Spiros and Hugo Geerts are employees of In Silico Biosciences.

\section{Disclosure}

The authors report no conflicts of interest in this work.

\section{References}

1. Kola I, Landis J. Can the pharmaceutical industry reduce attrition rates? Nat Rev Drug Discov. 2004;3(8):711-715.

2. Geerts H. Of mice and men: bridging the translational disconnect in CNS drug discovery. CNS Drugs. 2009;23(11):915-926.

3. Gibbs SE, D'Esposito M. A functional MRI study of the effects of bromocriptine, a dopamine receptor agonist, on component processes of working memory. Psychopharmacology (Berl). 2005;180(4):644-653.

4. Bilder RM, Volavka J, Lachman HM, Grace AA. The catechol-Omethyltransferase polymorphism: relations to the tonic-phasic dopamine hypothesis and neuropsychiatric phenotypes. Neuropsychopharmacology. 2004;29(11):1943-1961.

5. Bertolino A, Caforio G, Blasi G, et al. Interaction of COMT (Val(108/158)Met) genotype and olanzapine treatment on prefrontal cortical function in patients with schizophrenia. Am J Psychiatry. 2004;161(10):1798-1805

6. Weickert TW, Goldberg TE, Mishara A, et al. Catechol-O-methyltransferase val108/158 met genotype predicts working memory response to antipsychotic medications. Biol Psychiatry. 2004;56(9):677-682.

7. Slifstein M, Kolachana B, Simpson EH, et al. COMT genotype predicts cortical-limbic D1 receptor availability measured with [11C]NNC112 and PET. Mol Psychiatry. 2008;13(8):821-827.

8. Spiros A, Carr R, Geerts H. Not all partial dopamine $\mathrm{D}_{2}$ receptor agonists are the same in treating schizophrenia. Exploring the effects of bifeprunox and aripiprazole using a computer model of a primate striatal dopaminergic synapse. Neuropsychiatr Dis Treat. 2010;6:589-603.

9. Sautel F, Griffon N, Sokoloff P, et al. Nafadotride, a potent preferential dopamine D3 receptor antagonist, activates locomotion in rodents. J Pharmacol Exp Ther. 1995;275(3):1239-1246.

10. Sautel F, Griffon N, Levesque D, Pilon C, Schwartz JC, Sokoloff P. A functional test identifies dopamine agonists selective for D3 versus D2 receptors. Neuro Report. 1995;6(2):329-332.

11. Michaelides MR, Hong Y, DiDomenico S Jr, et al. (5aR,11bS)-4, 5,5a,6,7,11b-hexahydro-2-propyl-3-thia-5-azacyclopent-1-ena[c] phenanthrene-9,10-diol (A-86929): a potent and selective dopamine D1 agonist that maintains behavioral efficacy following repeated administration and characterization of its diacetyl prodrug (ABT-431). J Med Chem. 1995;38(18):3445-3447.

12. Yavich L, Forsberg MM, Karayiorgou M, Gogos JA, Mannisto PT. Site-specific role of catechol-O-methyltransferase in dopamine overflow within prefrontal cortex and dorsal striatum. J Neurosci. 2007;27(38): 10196-10209.

13. Montague PR, Hyman SE, Cohen JD. Computational roles for dopamine in behavioural control. Nature. 2004;431(7010):760-767.
14. Montague PR, McClure SM, Baldwin PR, et al. Dynamic gain control of dopamine delivery in freely moving animals. J Neurosci. 2004;24(7):1754-1759.

15. Castner SA, Goldman-Rakic PS, Williams GV. Animal models of working memory: insights for targeting cognitive dysfunction in schizophrenia. Psychopharmacology (Berl). 2004;174(1):111-125.

16. Cragg SJ, Hille CJ, Greenfield SA. Dopamine release and uptake dynamics within nonhuman primate striatum in vitro. J Neurosci. 2000; 20(21):8209-8217.

17. Doyle AE, Yager JD. Catechol-O-methyltransferase: effects of the val108 met polymorphism on protein turnover in human cells. Biochim Biophys Acta. 2008;1780(1):27-33.

18. Hirvonen MM, Nagren K, Rinne JO, et al. COMT Val158Met genotype does not alter cortical or striatal dopamine D2 receptor availability in vivo. Mol Imaging Biol. 2010;12(2):192-197.

19. Cho SS, Strafella AP. rTMS of the left dorsolateral prefrontal cortex modulates dopamine release in the ipsilateral anterior cingulate cortex and orbitofrontal cortex. PLoS ONE. 2009;4(8):e6725.

20. Okun I, Tkachenko SE, Khvat A, Mitkin O, Kazey V, Ivachtchenko AV. From anti-allergic to anti-Alzheimer's: molecular pharmacology of Dimebon. Curr Alzheimer Res. 2010;7(2):97-112.

21. Giorgetti M, Gibbons JA, Bernales S, et al. Cognition-enhancing properties of Dimebon in a rat novel object recognition task are unlikely to be associated with acetylcholinesterase inhibition or N-methyl-Daspartate receptor antagonism. J Pharmacol Exp Ther. 2010;333(3): $748-757$.

22. Tauscher J, Jones C, Remington G, Zipursky RB, Kapur S. Significant dissociation of brain and plasma kinetics with antipsychotics. $\mathrm{Mol}$ Psychiatry. 2002;7(3):317-321.

23. Hammarlund-Udenaes M. Active-site concentrations of chemicals - are they a better predictor of effect than plasma/organ/tissue concentrations? Basic Clin Pharmacol Toxicol. 2010;106(3):215-220.

24. Yokoi F, Grunder G, Biziere K, et al. Dopamine D2 and D3 receptor occupancy in normal humans treated with the antipsychotic drug aripiprazole (OPC 14597): a study using positron emission tomography and [11C]raclopride. Neuropsychopharmacology. 2002;27(2):248-259.

25. Jones RW. Dimebon disappointment. Alzheimers Res Ther. 2010; 2(5):25.

26. Yan M, Webster LT Jr, Blumer JL. Kinetic interactions of dopamine and dobutamine with human catechol-O-methyltransferase and monoamine oxidase in vitro. J Pharmacol Exp Ther. 2002;301(1):315-321.

27. Ho BC, Wassink TH, O’Leary DS, Sheffield VC, Andreasen NC. Catechol-O-methyl transferase Val158Met gene polymorphism in schizophrenia: working memory, frontal lobe MRI morphology and frontal cerebral blood flow. Mol Psychiatry. 2005;10(3):229,87-98.

28. Ramos BP, Arnsten AF. Adrenergic pharmacology and cognition: focus on the prefrontal cortex. Pharmacol Ther. 2007;113(3):523-536.

29. Van der Schyf CJ. The use of multi-target drugs in the treatment of neurodegenerative diseases. Expert Rev Clin Pharmacol. 2011;4(3): 293-298.

30. Wong EH, Nikam SS, Shahid M. Multi- and single-target agents for major psychiatric diseases: therapeutic opportunities and challenges. Curr Opin Investig Drugs. 2008;9(1):28-36.
Journal of Experimental Pharmacology

\section{Publish your work in this journal}

The Journal of Experimental Pharmacology is an international, peerreviewed, open access journal publishing original research, reports, reviews and commentaries on all areas of laboratory and experimental pharmacology. The manuscript management system is completely online and includes a very quick and fair peer-review system.

\section{Dovepress}

Submit your manuscript here: http://www.dovepress.com/journal-of-experimental-pharmacology-journal 